\title{
Natural inflation with natural trans-planckian axion decay constant from anomalous $\mathrm{U}(1)_{X}$
}

\author{
Tianjun Li, ${ }^{a, b}$ Zhijin $\mathbf{L i}^{c}$ and Dimitri V. Nanopoulos ${ }^{c, d, e}$ \\ ${ }^{a}$ State Key Laboratory of Theoretical Physics \\ and Kavli Institute for Theoretical Physics China (KITPC), \\ Institute of Theoretical Physics, Chinese Academy of Sciences, Beijing 100190, P.R. China \\ ${ }^{b}$ School of Physical Electronics, University of Electronic Science and Technology of China, \\ Chengdu 610054, P.R. China \\ ${ }^{c}$ George P. and Cynthia W. Mitchell Institute for Fundamental Physics and Astronomy, \\ Texas A $6 M$ University, College Station, TX 7r843, U.S.A. \\ ${ }^{d}$ Astroparticle Physics Group, Houston Advanced Research Center (HARC), \\ Mitchell Campus, Woodlands, TX 77381, U.S.A. \\ ${ }^{e}$ Academy of Athens, Division of Natural Sciences, \\ 28 Panepistimiou Avenue, Athens 10679, Greece \\ E-mail: tli@itp.ac.cn, lizhijin@physics.tamu.edu, \\ dimitri@physics.tamu.edu
}

ABSTRACT: We propose a natural inflation model driven by an imaginary or axionic component of a Kähler modulus in string-inspired supergravity. The shift symmetry of the axion is gauged under an anomalous $\mathrm{U}(1)_{X}$ symmetry, which leads to a modulus-dependent FayetIliopoulos (FI) term. The matter fields are stabilized by F-terms, and the real component of the modulus is stabilized by the $\mathrm{U}(1)_{X} \mathrm{D}$-term, while its axion remains light. Therefore, the masses of real and imaginary components of the modulus are separated at different scales. The scalar potential for natural inflation is realized by the superpotential from the non-perturbative effects. The trans-Planckian axion decay constant, which is needed to fit with BICEP2 observations, can be obtained naturally in this model.

KEYwords: Cosmology of Theories beyond the SM, Supergravity Models, Anomalies in Field and String Theories

ARXIV EPRINT: 1405.1804 


\section{Contents}

1 Introduction 1

2 Natural inflation model building 2

3 Matter fields and modulus stabilization 4

3.1 Matter fields stabilization 4

$\begin{array}{lll}3.2 & \text { F-term potential after stabilization } & 7\end{array}$

$\begin{array}{ll}3.3 & \text { Anomalous } \mathrm{U}(1)_{X} \text { D-term and modulus stabilization }\end{array}$

$\begin{array}{llr}4 & \text { Natural inflation potential } & 8\end{array}$

5 Conclusion 10

\section{Introduction}

The recent discovery of the B-mode polarization by the BICEP2 Collaboration, if confirmed, provides further strong evidence on the inflationary paradigm for the early epoch of the Universe [1]. The observed tensor-to-scalar ratio $r$ is $r=0.20_{-0.05}^{+0.07}$, or $r=0.16_{-0.05}^{+0.06}$ without the dust contributions. One of the inflation models, which agrees with the BICEP2 results, is the well-known natural inflation $[2,3]$.

The motivation of natural inflation $[2,3]$ is to solve the flatness problem of inflation potential at tree level, and remains flat against radiative corrections. The continuous shift symmetry protects the flatness of inflation potential. To realize inflation, the continuous symmetry should be broken to a discrete shift symmetry $\phi \rightarrow \phi+2 \pi f$ with $f$ the axion (or inflaton) decay constant, and the potential for natural inflation is

$$
V(\phi)=\Lambda^{4}\left(1 \pm \cos \left(\frac{\phi}{f}\right)\right)
$$

where $\Lambda$ is the inflation energy scale around $2 \times 10^{16} \mathrm{GeV}$ or $10^{-2} \mathrm{M}_{\mathrm{Pl}}$ for $r=0.16 / 0.20$ with $\mathrm{M}_{\mathrm{Pl}}$ the reduced Planck mass $\left(\mathrm{M}_{\mathrm{Pl}}=2.4 \times 10^{18} \mathrm{GeV}\right)$.

Axion is a "natural" inflaton candidate since it preserves the exact continuous shift symmetry at perturbative level. Axions can be obtained from antisymmetric tensor fields in string theory through spacetime compactification [4]. Considering the non-perturbative effects, such as gaugino condensation or instanton effect, one can break such continuous shift symmetry of axion to the discrete symmetry and then realize natural inflation in string theory.

The challenge to natural inflation is the large decay constant $f$. To generate sufficient large tensor fluctuations that are consistent with BICEP2 observations, the decay constant 
$f$ should be trans-Planckian $f \sim O(10)$ in the Planck units [5]. However, in string theory the axion decay constant cannot be larger than the string scale $\mathrm{M}_{\text {String }}[4,6,7]$, which is about one order below the reduced Planck scale as required by the weak interaction assumption. An effective large axion decay constant was realized by the N-flation $[8,9]$ or the aligned axion mechanism [10]. In the aligned natural inflation [10], two axions with small decay constants are carefully adjusted to form a flat direction for inflation, and then one-linear combination of two axions can have the effective trans-Planckian decay constant. Recently, there are many works proposed to realize large decay constant with multi-aligned axions [11-14] or realize N-flation and natural inflation in string theory [15-21].

To obtain inflation in string theory or its low energy approximation-supergravity (SUGRA), there is a general problem on the moduli stabilization. For single field inflation, all the scalars except the inflaton should be fixed during inflation. The well-known KKLT mechanism based on F-term was proposed in [22], where the complex-structure moduli are stabilized by the fluxes [23] while the Kähler modulus is stabilized through the non-perturbative effects. The difficulty to realize axion inflation based on the KKLT mechanism is: once the real component of the Kähler modulus is fixed, its axionic component obtains large mass as well and then destroys inflation [24, 25]. This problem can be solved by considering the modulus-dependent FI term associated with anomalous $\mathrm{U}(1)_{X}[26]$. The FI term only depends on the real component of modulus, which can obtain large mass from D-term flatness. The axion is still light as it decouples from D-term.

In this work, we construct a natural inflation model in string-inspired SUGRA where only one modulus couples to the matter fields. The shift symmetry of axion is gauged to obtain anomalous $\mathrm{U}(1)_{X}$. The gauge invariant superpotential consists of the non-perturbative term of modulus and various couplings among matter fields. All the matter fields are stabilized by F-terms, and the vanishing D-term gives large mass to the real component of the modulus. The axion is still light after modulus stabilization and its potential is given exactly by eq. (1.1). Besides, the trans-Planckian axion decay constant can be obtained naturally by taking the large condensation gauge group and numbers of $\mathrm{U}(1)_{X}$ charged matter fields (around 20).

This paper is organized as follows. In section 2 we provide the string-inspired SUGRA structure for model building and show the anomaly cancellation of $\mathrm{U}(1)_{X}$. In section 3 the matter fields/modulus stabilizations based on the F-terms/D-term are discussed. In section 4 we obtain the natural inflation potential after stabilizations, and the transPlanckian axion decay constant is realized from the non-perturbative effects with suitable condensation gauge group. We conclude in section 5 .

\section{Natural inflation model building}

We consider the following Kähler potential

$$
K=-\ln (T+\bar{T})+\phi_{i} \bar{\phi}_{i}+\chi_{j} \bar{\chi}_{j}+\varphi_{k} \bar{\varphi}_{k}+Q \bar{Q}+X_{l} \bar{X}_{l},
$$

in which the indexes are $i=1,2, \cdots, m, j=1,2, \cdots, m-2, k=1,2,3,4$ and $l=$ $0,1,2, \cdots, m$. The modulus $T$ can be dilaton superfield or one of the Kähler moduli from 
string compactification. The $\mathrm{U}(1)_{X}$ charged matter fields $\left(\phi_{i}, \chi_{j}, \varphi_{k}, Q\right)$, which will be generically denoted as $z_{h}$, are introduced to construct gauge invariant superpotential and cancel the gauge anomalies. The modulus $T$ and matter fields $z_{h}$ transform under $\mathrm{U}(1)_{X}$ as follows

$$
\begin{aligned}
T & \rightarrow T+i \delta \epsilon \\
z_{h} & \rightarrow z_{h} e^{i \epsilon q_{z_{h}}},
\end{aligned}
$$

in which the $\mathrm{U}(1)_{X}$ charges $q_{z}$ are $q_{\phi_{i}}=-q_{\chi_{j}}=q, q_{\varphi_{k}}=(-1)^{k} q, q_{Q}=-2 q . X_{l}$ are neutral under $\mathrm{U}(1)_{X}$ and vanish during inflation, their F-terms are used to stabilize $\phi_{i}$ and $\varphi_{1,2}$ with non-zero vacuum expectation values (VEV).

The superpotential is

$$
\begin{aligned}
W= & w_{0}+a \phi_{1}^{\frac{1}{n}} e^{-b T}+X_{i}\left(\phi_{i} \varphi_{1}-\lambda_{1}\right)+X_{0}\left(\varphi_{1} \varphi_{2}-\lambda_{2}\right) \\
& +c_{1}\left(\varphi_{1} \varphi_{4}+\varphi_{2} \varphi_{3}\right)+m_{j} \varphi_{4} \chi_{j}+\varphi_{4}^{2} Q .
\end{aligned}
$$

Given $q=n b \delta$, above superpotential is obviously $\mathrm{U}(1)_{X}$ invariant. The first two terms in (2.3) is similar with the KKLT scenario in a gauge invariant form [22]. The constant term $w_{0}$ is from the complex-structure moduli stabilization. Different from the KKLT scenario, the non-perturbative term $a \phi_{1}^{\frac{1}{n}} e^{-b T}$ is $\mathrm{U}(1)_{X}$ invariant. The $\mathrm{U}(1)_{X}$ transformation of matter field $\phi_{1}^{1 / n} \rightarrow \phi_{1}^{1 / n} e^{i q / n}$ cancels the phase factor $e^{-i b \delta}$ from shift of the modulus $T \rightarrow T+i \epsilon \delta$ under $\mathrm{U}(1)_{X}$. Such kind of non-perturbative superpotential can be obtained from the gaugino condensation with massive chiral superfields, which form representation of the condensation group, such as $\mathrm{SU}(n)$ and can be integrated out in effective field theory. The effective superpotential is guaranteed to be $\mathrm{U}(1)_{X}$ invariant $[27-29,32]$. Effects of the gauge invariant non-perturbative term on moduli stabilization and inflation have been studied in [30-35]. Furthermore, in this form the matter field $\phi_{1}$ has positive exponent, and gives an analytic coefficient for the non-perturbative term, which makes the anomalous $\mathrm{U}(1)_{X}$ D-term cancellable. The D-term flatness is needed for modulus stabilization.

In eq. (2.3), we will have many other superpotential terms, for example, $\phi_{i} \chi_{j}$, etc, which are allowed by the $\mathrm{U}(1)_{X}$ symmetry but neglected. To solve this problem, we can introduce a $Z_{m}$ discrete symmetry under which $\varphi_{3}, \varphi_{4}, \chi_{j}$, and $Q$ transform as follows

$$
\varphi_{3} \rightarrow \omega^{k} \varphi_{3}, \varphi_{4} \rightarrow \omega^{-k} \varphi_{4}, \chi_{j} \rightarrow \omega^{k} \chi_{j}, Q \rightarrow \omega^{2 k} Q
$$

with $\omega^{m}=1$, while all the other fields are neutral under $Z_{m}$. Thus, superpotential will be

$$
\begin{aligned}
W= & w_{0}+a \phi_{1}^{\frac{1}{n}} e^{-b T}+X_{i}\left(\phi_{i} \varphi_{1}-\lambda_{1}\right)+X_{0}\left(\varphi_{1} \varphi_{2}-\lambda_{2}\right) \\
& +c_{1} \varphi_{1} \varphi_{2}+m_{0} \varphi_{3} \varphi_{4}+m_{j} \varphi_{4} \chi_{j}+\varphi_{4}^{2} Q .
\end{aligned}
$$

With our numerical study assumption, we will point out that the superpotentials in both eq. (2.3) and eq. (2.5) will give the similar inflaton potential. In general, we can have the superpotential terms $c_{i}^{\prime} \phi_{i} \varphi_{1}+c_{1} \varphi_{1} \varphi_{2}$. Without loss of generality, we can make a tranformation for $\phi_{i} / \varphi_{2}$, and $X_{i}$, and obtain the above superpotential since $\varphi_{1}$ is only coupled to one linear combination of $\phi_{i}$ and $\varphi_{2}$. 
Gauge anomaly cancellation. The anomalous $\mathrm{U}(1)_{X}$ plays a special role in the quantum anomaly cancellation through the Green-Schwarz mechanism in four dimensional spacetime [36]. The gauge kinetic term of $\mathrm{U}(1)_{X}$ is

$$
\int d^{2} \theta f W_{\alpha}^{2}
$$

in which $W_{\alpha}$ is $\mathrm{U}(1)_{X}$ gauge field strength. Here we take the gauge kinetic function $f=k_{X} T$. The gauge kinetic term contains two parts $\operatorname{Re}(f) F^{2}$ and $\operatorname{Im}(f) F \tilde{F}$. The first term is $\mathrm{U}(1)_{X}$ invariant, while the second term transforms non-trivially under $\mathrm{U}(1)_{X}$. The shift of modulus $T$ introduces an extra term $i \delta k_{X} \int d^{2} \theta W_{\alpha}^{2}$, which cancels the anomaly from charged fermionic fields and keep the theory anomaly free.

Ignoring the anomaly of condensation gauge group $\mathrm{SU}(n)$, we need to consider two kinds of anomalies: the gravitational anomaly $\mathrm{U}(1)_{X}$ and the cubic anomaly $\mathrm{U}(1)_{X}^{3}$. The fermionic contributions are:

$$
\begin{aligned}
& \operatorname{Tr} q_{z}=\sum_{z} q_{z}=0, \\
& \operatorname{Tr} q_{z}^{3}=\sum_{z} q_{z}^{3}=-6 q^{3} .
\end{aligned}
$$

The gravitational anomaly is canceled without higher derivative terms $R^{2}$. Anomaly cancelation of cubic term $\mathrm{U}(1)_{X}^{3}$ requires

$$
k_{X} \delta=-\frac{1}{48 \pi^{2}} \sum_{z} q_{z}^{3}=\frac{1}{8 \pi^{2}} q^{3} .
$$

As $q=n b \delta$, we have $k_{X}=n b q^{2} / 8 \pi^{2}$.

\section{Matter fields and modulus stabilization}

In this model, the matter fields are stabilized by F-terms. Even though there is the KKLT type superpotential in (2.3), it has nothing to do with modulus stabilization but provides potential for natural inflation, actually, the real component of the modulus is stabilized by $\mathrm{U}(1)_{X}$ D-term.

The F-term scalar potential is given by

$$
V_{F}=e^{K}\left(K^{i \bar{j}} D_{i} W D_{\bar{j}} \bar{W}-3 W \bar{W}\right),
$$

in which $K^{i \bar{j}}$ is the inverse of the Kähler metric $K_{i \bar{j}}=\partial_{i} \partial_{\bar{j}} K$ and $D_{i} W=W_{i}+K_{i} W$. The complete expression of $V_{F}$ is rather tedious, nevertheless, it can be remarkably simplified after field stabilization.

\subsection{Matter fields stabilization}

For matter fields stabilization, we ignore the constant term and the non-perturbative term, as they provide inflationary potential which is significantly lower than the matter fields stabilization scale. Their effects will be estimated later. 
Clearly the matter fields $\chi_{j}, \varphi_{3,4}, Q$ and $X_{l}$ have global minimum at origin while extra matter fields $\phi_{i}$ and $\varphi_{1,2}$ will get non-trivial VEVs. During inflation they will evolve to the minimum rapidly driven by the exponential factor $e^{K}$ of F-term scalar potential and the large masses obtained from the matter couplings in eq. (2.3). Therefore the value of superpotential during inflation is simplified as $\langle W\rangle \equiv W_{0}=w_{0}+a \phi_{1}^{\frac{1}{n}} e^{-b T}$. In eq. (3.1), only these terms independent with $\chi_{j}, \varphi_{3,4}, Q$ and $X_{l}$ are non-vanishing. The non-vanishing F-terms $V_{z}$ introduced by fields $z$ are (multiplied by an overall factor $e^{K}$ ):

- for $T$ :

$$
\begin{gathered}
V_{T}=(T+\bar{T})^{2}\left[a^{2} b^{2}\left(\phi_{1} \bar{\phi}_{1}\right)^{\frac{1}{n}} e^{-b(T+\bar{T})}+2 a^{2} b \frac{1}{T+\bar{T}}\left(\phi_{1} \bar{\phi}_{1}\right)^{\frac{1}{n}} e^{-b(T+\bar{T})}\right. \\
\left.+a b w_{0} \frac{1}{T+\bar{T}}\left(\phi^{\frac{1}{n}} e^{-b T}+\bar{\phi}^{\frac{1}{n}} e^{-b \bar{T}}\right)\right]+W_{0} \bar{W}_{0},
\end{gathered}
$$

- for $\phi_{1}$ :

$$
\begin{aligned}
V_{\phi_{1}}= & \frac{a^{2}}{n^{2}}\left(\phi_{1} \bar{\phi}_{1}\right)^{\frac{1}{n}-1} e^{-b(T+\bar{T})}+\frac{2 a^{2}}{n}\left(\phi_{1} \bar{\phi}_{1}\right)^{\frac{1}{n}} e^{-b(T+\bar{T})} \\
& +\frac{a}{n} w_{0}\left(\phi^{\frac{1}{n}} e^{-b T}+\bar{\phi}^{\frac{1}{n}} e^{-b \bar{T}}\right)+\phi_{1} \bar{\phi}_{1} W_{0} \bar{W}_{0},
\end{aligned}
$$

- for $\phi_{i>1}$ :

$$
V_{\phi_{i>1}}=\phi_{i>1} \bar{\phi}_{i>1} W_{0} \bar{W}_{0}
$$

- and similarly, for $\varphi_{1}$ and $\varphi_{2}$ :

$$
V_{\varphi_{1,2}}=(\varphi \bar{\varphi})_{1,2} W_{0} \bar{W}_{0}
$$

Above terms are proportional to $a^{2}, a w_{0}$ or $w_{0}^{2}$. In this model, the parameters have orders of $a \sim w_{0} \sim 10^{-5}$, while $c_{1} \sim 10^{-3}-10^{-2}$, therefore above terms are extremely small comparing with the following F-terms.

For the superpotential in eq. (2.5), $V_{T}, V_{\phi_{1}}, V_{\phi_{i>1}}$ are the same, while $V_{\varphi_{1,2}}$ become

$$
V_{\varphi_{1,2}}=\left|\bar{\varphi} W_{0}+c_{1} \varphi_{2,1}\right|^{2} \text {. }
$$

Note that in the above equation only the terms $\left|c_{1} \varphi_{1,2}\right|^{2}$ are relevant while all the other terms are small and negligible, thus, all the rest discussions will be the same and we will not repeat here.

The vanishing neutral matter fields $X_{l}$ provide F-terms $\left|W_{X_{l}}\right|^{2}$ in the scalar potential, besides, $\varphi_{1}$ and $\varphi_{2}$ get mass terms from $\left|W_{\varphi_{4}}\right|^{2}$ and $\left|W_{\varphi_{3}}\right|^{2}$, respectively. Combining these F-terms together we have

$$
V_{F_{1}}=e^{K}\left|W_{X_{l}}\right|^{2}=e^{K}\left(\sum_{i}\left|\phi_{i} \varphi_{1}-\lambda_{1}\right|^{2}+\left|\varphi_{1} \varphi_{2}-\lambda_{2}\right|^{2}+c_{1}^{2}\left(\left|\varphi_{1}\right|^{2}+\left|\varphi_{2}\right|^{2}\right)\right) .
$$

Of course there are numerous corrections containing $\phi_{i}$ and $\varphi_{1,2}$ in (3.2), (3.3), (3.4), (3.5), however, they are either significantly smaller than (3.7) or can be canceled with each other. The VEVs of $\phi_{i}$ are mainly dominated by the results obtained from (3.7). 
Taking $\varphi_{i}=r_{i} e^{i \theta_{i}}$, the potential (3.7) becomes

$$
\begin{aligned}
V_{F_{1}} & =e^{K}\left|W_{X_{l}}\right|^{2}=e^{K}\left\{\sum_{i}\left|\phi_{i} \varphi_{1}-\lambda_{1}\right|^{2}+r_{1}^{2} r_{2}^{2}-2 \lambda_{2} r_{1} r_{2} \cos \left(\theta_{1}+\theta_{2}\right)+\lambda_{2}^{2}+c_{1}^{2}\left(r_{1}^{2}+r_{2}^{2}\right)\right\} \\
& \geqslant e^{K}\left\{\sum_{i}\left|\phi_{i} \varphi_{1}-\lambda_{1}\right|^{2}+r_{1}^{2} r_{2}^{2}-2 \lambda_{2} r_{1} r_{2}+\lambda_{2}^{2}+c_{1}^{2}\left(r_{1}^{2}+r_{2}^{2}\right)\right\} \\
& =e^{K}\left\{\sum_{i}\left|\phi_{i} \varphi_{1}-\lambda_{1}\right|^{2}+\left(r_{1} r_{2}-\left(\lambda_{2}-c_{1}^{2}\right)\right)^{2}+c_{1}^{2}\left(r_{1}-r_{2}\right)^{2}+c_{1}^{2}\left(2 \lambda_{2}-c_{1}^{2}\right)\right\} .
\end{aligned}
$$

The vacuum takes place at $\theta_{1}+\theta_{2}=2 n \pi$, while the direction $\theta_{1}-\theta_{2}$ is flat. The minimization of the potential in the bracket gives

$$
\begin{aligned}
r_{1} & =r_{2}=r_{0}=\sqrt{\lambda_{2}-c_{1}^{2}}, \\
\left|\phi_{i}\right| & \equiv r=\frac{\lambda_{1}}{r_{0}} .
\end{aligned}
$$

The orders of parameters are simply taken as $r_{0} \sim 10^{-2}$ and $r^{2}=0.1$ in following estimations. Eq. (3.8) provides strong stabilization on the matter fields. For the condition $r_{1}=r_{2}$, the mass of direction along $\left(r_{1}-r_{2}\right) / \sqrt{2}$ is $2 c_{1} \sim 10^{-3}-10^{-2} \gg H$, where $H$ is Hubble constant during inflation, therefore it is strongly stabilized as well. Besides, we get a cosmology constant term $V_{0}=c_{1}^{2}\left(2 \lambda_{2}-c_{1}^{2}\right)$.

The anomalous $\mathrm{U}(1)_{X}$ is spontaneously broken by VEVs of charged matter fields. The phases of $\phi_{i}=r e^{i \alpha_{i}}$ satisfy $\alpha_{i}=\theta_{2}$, we take the $\mathrm{U}(1)_{X}$ gauge $\theta_{2}=0$ for simplicity. The flat direction is absorbed by $\mathrm{U}(1)_{X}$ massive vector field through the Higgs mechanism after spontaneous symmetry broken.

It should be careful to consider above values as VEVs of these fields. Actually the cosmology constant term $V_{0}$, together with the overall factor $e^{K}$, can shift the non-zero VEVs. Specifically, the first condition $r_{1}=r_{2}$ remains the same, while the results of $\phi_{i}$ and $r_{0}=\sqrt{\lambda_{2}-c_{1}^{2}}$ will be slightly modified.

In general, considering a field $\phi$ with mass $m_{0}$ and non-zero VEV $\phi_{0}$, assuming its Kähler potential is minimal, and the overall scalar potential $V=e^{\phi^{2}}\left(\frac{1}{2} m_{0}^{2}\left(\phi-\phi_{0}\right)^{2}+V_{0}\right)$, where $V_{0}$ is the residual cosmology constant term after field stabilization or during inflation, the vacuum is determined by

$$
\frac{d V}{d \phi}=e^{\phi^{2}}\left(m_{0}^{2}\left(\phi-\phi_{0}\right)+m_{0}^{2} \phi\left(\phi-\phi_{0}\right)^{2}+2 V_{0} \phi\right)=0,
$$

which gives a new VEV $\phi_{0}^{\prime} \simeq \phi_{0}-2 V_{0} \phi_{0} / m_{0}^{2}$. During inflation, the quasi-cosmology constant provided by inflaton is $V_{0} \sim 10^{-8}$ in the Planck units, $m_{0}$ is of order $O\left(10^{-2}\right)$ in our model, therefore the shift of matter fields is about $\Delta \phi / \phi_{0} \simeq 10^{-4}$. The vacuum energy is reduced by $2 V_{0}^{2} \phi_{0}^{2} / m_{0}^{2} \sim 10^{-13}$, which is completely ignorable during inflation. Therefore we can safely consider the values obtained in (3.9) are VEVs of these fields. Besides, there is a coupling between $\phi_{1}$ and modulus $T$ through $a \phi_{1}^{\frac{1}{n}} e^{-b T}$, however, we will show that the interaction is seriously suppressed by $a w_{0} \sim 10^{-9}$ and has ignorable effect on $\phi_{1}$ stabilization as well. 


\section{2 $\quad$ F-term potential after stabilization}

After field stabilization, the F-term potential is simplified. Besides an overall factor $e^{K}$, it is

$$
\begin{aligned}
V= & (T+\bar{T})^{2}\left[a^{2} b^{2}\left(\phi_{1} \bar{\phi}_{1}\right)^{\frac{1}{n}} e^{-b(T+\bar{T})}+2 a^{2} b \frac{1}{T+\bar{T}}\left(\phi_{1} \bar{\phi}_{1}\right)^{\frac{1}{n}} e^{-b(T+\bar{T})}\right. \\
& \left.+a b w_{0} \frac{1}{T+\bar{T}}\left(\phi_{1}^{\frac{1}{n}} e^{-b T}+\bar{\phi}_{1}^{\frac{1}{n}} e^{-b \bar{T}}\right)\right]+\frac{a^{2}}{n^{2}}\left(\phi_{1} \bar{\phi}_{1}\right)^{\frac{1}{n}-1} e^{-b(T+\bar{T})} \\
& +\frac{2 a^{2}}{n}\left(\phi_{1} \bar{\phi}_{1}\right)^{\frac{1}{n}} e^{-b(T+\bar{T})}+\frac{a}{n} w_{0}\left(\phi_{1}^{\frac{1}{n}} e^{-b T}+\bar{\phi}_{1}^{\frac{1}{n}} e^{-b \bar{T}}\right) \\
& +c_{1}^{2}\left(2 \lambda_{2}-c_{1}^{2}\right)+\left(\sum_{i}\left|\phi_{i}\right|^{2}+\left|\varphi_{1}\right|^{2}+\left|\varphi_{2}\right|^{2}-2\right) W_{0} \bar{W}_{0} .
\end{aligned}
$$

Taking $r_{0}^{2} \ll r^{2}$, we ignore the contributions from $\left|\varphi_{1,2}\right|^{2} W_{0} \bar{W}_{0}$. The parameters can be simply taking as $\sum_{i}\left|\phi_{i}\right|^{2}=m r^{2}=2$ so that the last term in eq. (3.11) vanishes.

Couplings between modulus $T$ and $\phi_{1}$ are shown in eq. (3.11), the dominant term is $g(T) \phi_{1}^{\frac{1}{n}}$ with $g(T) \sim 10^{-8}$. Ignoring the factor $e^{K}$, the potential of $\phi_{1}$ is approximate to

$$
V_{\phi_{1}}=\frac{1}{2} m_{1}^{2}\left(\phi_{1}-r\right)^{2}+g(T) \phi_{1}^{\frac{1}{n}}
$$

in which $m_{1}^{2} \sim 10^{-4}$. VEV of $\phi_{1}$ is shifted about $\Delta\left\langle\phi_{1}\right\rangle \simeq g(T) r^{\frac{1}{n}} / n m_{1}^{2} r \sim 10^{-4}$, and the energy is reduced by $\frac{1}{2} \frac{g(T)^{2} r^{2 / n}}{n^{2} m_{1}^{2} r^{2}} \sim 10^{-12}$, which confirms that the non-perturbative effect is ignorable for matter field stabilization.

\subsection{Anomalous $\mathrm{U}(1)_{X}$ D-term and modulus stabilization}

The anomalous $\mathrm{U}(1)_{X} \mathrm{D}$-term scalar potential is given by

$$
V_{D}=\frac{1}{2 \operatorname{Re}(f)} D^{2}
$$

where $D=i K_{z} X^{z}+i \frac{W_{z}}{W} X^{z} . X^{z}$ are the components of Killing vector corresponding to $\mathrm{U}(1)_{X}$ isometries of the Kähler manifold, which are

$$
\left(X^{T}, X^{\phi_{i}}, X^{\chi_{j}}, X^{\varphi_{k}}, X^{Q}\right)=\left(i \delta, i q \phi_{i},-i q \chi_{j},(-1)^{k} i q \varphi_{k},-2 i q Q\right) .
$$

In this model the superpotential is gauge invariant, the $\mathrm{D}$-term is simplified as $D=i K_{z} X^{z}$ and it reads

$$
D=\frac{\delta}{T+\bar{T}}-q \sum_{i}\left|\phi_{i}\right|^{2}+q \sum_{j}\left|\chi_{j}\right|^{2}-(-1)^{k} q \sum_{k}\left|\varphi_{k}\right|^{2}+2 q|Q|^{2} .
$$

After field stabilization, $\chi_{j}, \varphi_{3,4}$ and $Q$ have vanshing VEVs, the $\varphi_{1}$ and $\varphi_{2}$ D-terms cancel each other due to $\left|\varphi_{1}\right|=\left|\varphi_{2}\right|$, and then the D-term is reduced to

$$
D=\frac{\delta}{T+\bar{T}}-q \sum_{i}\left|\phi_{i}\right|^{2}=\frac{\delta}{T+\bar{T}}-m q r^{2} .
$$


The D-term potential is

$$
\begin{aligned}
V_{D} & =\frac{1}{2 k_{X} T_{R}}\left(\frac{\delta}{T+\bar{T}}-m q r^{2}\right)^{2} \\
& =\frac{4 \pi^{2}}{(n b)^{3} T_{R}}\left(\frac{1}{2 T_{R}}-m n b r^{2}\right)^{2},
\end{aligned}
$$

in which $T_{R} \equiv \operatorname{Re} T$ and the gauge invariant condition $q=n b \delta$ and cubic anomaly $\mathrm{U}(1)_{X}^{3}$ cancelation $k_{X}=n b q^{2} / 8 \pi^{2}$ are used. The D-term vanishing condition gives $\left\langle T_{R}\right\rangle \equiv$ $T_{0}=1 / 2 m n b r^{2}$. In the simplified case with $m r^{2}=2$, the real component of modulus is $T_{0}=1 / 4 n b$.

\section{Natural inflation potential}

We have stabilized all the fields except the imaginary component of $T$. Now the scalar potential (3.11) becomes

$$
\begin{aligned}
V=2 e^{2} b\left[a^{2} r^{\frac{2}{n}} e^{-\frac{1}{2 n}}\right. & \left(\frac{1}{4 n}+3+\frac{1}{n r^{2}}\right) \\
& \left.+n c_{1}^{2}\left(2 \lambda_{2}-c_{1}^{2}\right)+3 a w_{0} r^{\frac{1}{n}} e^{-\frac{1}{4 n}} \cos (b \theta)\right],
\end{aligned}
$$

where $\theta$ is the imaginary component of $T$. To get global Minkowski vacuum the parameters need to be adjusted so that

$$
n c_{1}^{2}\left(2 \lambda_{2}-c_{1}^{2}\right)+a^{2} r^{\frac{2}{n}} e^{-\frac{1}{2 n}}\left(\frac{1}{4 n}+3+\frac{1}{n r^{2}}\right)=3 a w_{0} r^{\frac{1}{n}} e^{-\frac{1}{4 n}}
$$

and we get the scalar potential

$$
V=6 a b w_{0} e^{2} r^{\frac{1}{n}} e^{-\frac{1}{4 n}}(1+\cos (b \theta))
$$

which is of the same form in eq. (1.1) with $\Lambda^{4}=6 a b w_{0} e^{2} r^{1 / n} e^{-1 / 4 n}$. Taking $a \simeq 5 \times 10^{-5}$, $w_{0} \sim 6 \times 10^{-5}, r^{2} \sim 0.1, b=0.1$ and $n \geqslant 6$, it gives the inflation scale $\Lambda \sim 10^{-2}$ in the Planck units.

Before we consider $\theta$ as our inflaton, a field re-scale is needed to get canonical kinetic term, and this will affect the decay constant of $\theta$.

The kinetic term of $T$ is

$$
L_{K}=\frac{1}{(T+\bar{T})^{2}} \partial_{\mu} T \partial^{\mu} T=\frac{1}{4 T_{0}^{2}}\left(\partial_{\mu} T_{R} \partial^{\mu} T_{R}+\partial_{\mu} \theta \partial^{\mu} \theta\right) .
$$

Defining $\theta=\sqrt{2} T_{0} \rho$, we get the action of inflaton

$$
L=\frac{1}{2} \partial_{\mu} \rho \partial^{\mu} \rho+\Lambda^{4}\left(1+\cos \left(\frac{\rho}{\sqrt{2} m n r^{2}}\right)\right)
$$

in which the D-term stabilization condition $b T_{0}=1 / 2 m n r^{2}$ has been used. The axion decay constant in this model is $f=\sqrt{2} m n r^{2}$. The VEV of matter fields $r$ is smaller than 
the Planck mass. Without uplift from parameters $m, n$, the axion decay constant $f$ cannot be super-Planckian. Nevertheless, $f$ is proportional to the product of charged field number $m$ and the degree of condensation gauge group $n$, it is very easy to get super-Plankian $f$ by taking large $m$ or $n$. We used $r^{2}=0.1$ and $m r^{2}=2$ before, in such case we have $m=20$, then $f=2 \sqrt{2} n$ and it is of order $O(10)$ with $n=4$. By using larger $n$ the axion decay constant $f$ increases linearly, the potential gets close to the type $\frac{1}{2} m^{2} \rho^{2}$, and we get chaotic inflation.

Gravitino mass. Even though the matter fields are stabilized at the scales much higher than inflation scale, they do not introduce too heavy gravitino mass. After field stabilization, the pure matter couplings in the superpotential in eq. (2.3) vanish, the VEV of superpotential $\langle W\rangle$ during inflation is

$$
\langle W\rangle \equiv W_{0}=w_{0}+a\left\langle\phi_{1}^{\frac{1}{n}} e^{-b T}\right\rangle=w_{0}-a r^{\frac{1}{n}} e^{-\frac{1}{4 n}}
$$

Besides, we also have the VEV of $e^{K}$

$$
\left\langle e^{K}\right\rangle=\frac{e^{2}}{2 T_{0}}
$$

in which we have used $m r^{2}=2$ and the small term $2 r_{1}^{2}$ from VEVs of $\varphi_{1,2}$ is ignored. The gravitino mass is

$$
\begin{aligned}
M_{\frac{3}{2}} \equiv \exp \left(\frac{\langle G\rangle}{2}\right) & =\left\langle e^{\frac{K}{2}}\left(W_{0} \bar{W}_{0}\right)^{\frac{1}{2}}\right\rangle \\
& =e \sqrt{2 n b}\left(w_{0}-a r^{\frac{1}{n}} e^{-\frac{1}{4 n}}\right) .
\end{aligned}
$$

The gravitino mass relates to the inflation energy scale through

$$
(e \sqrt{2 n b})^{2} w_{0} a r^{\frac{1}{n}} e^{-\frac{1}{4 n}}=\frac{n}{3} \Lambda^{4} .
$$

Small gravitino mass can be obtained by taking $w_{0} \rightarrow a r^{\frac{1}{n}} e^{-\frac{1}{4 n}}$, however, eq. (4.2) provides a lower bound on it

$$
\begin{aligned}
w_{0}-a r^{\frac{1}{n}} e^{-\frac{1}{4 n}} & =n c_{1}^{2}\left(2 \lambda_{2}-c_{1}^{2}\right)\left(3 a r^{\frac{1}{n}} e^{-\frac{1}{4 n}}\right)^{-1}+\frac{1}{3 n} a r^{\frac{1}{n}} e^{-\frac{1}{4 n}}\left(\frac{1}{4}+\frac{1}{r^{2}}\right) \\
& \geqslant \frac{2}{3} c_{1}\left(2 \lambda_{2}-c_{1}^{2}\right)^{\frac{1}{2}}\left(\frac{1}{4}+\frac{1}{r^{2}}\right)^{\frac{1}{2}} .
\end{aligned}
$$

The minimum locates at $c_{1}^{2}\left(2 \lambda_{2}-c_{1}^{2}\right) \simeq\left(\frac{1}{4}+\frac{1}{r^{2}}\right) w_{0}^{2} / n^{2}$. For $c_{1} \sim 10^{-3}, \lambda_{2} \sim 10^{-4}$, the gravitino mass can be reduced to the order of $10^{-5}$, while it cannot get significantly smaller otherwise the field stabilization is not strong enough for inflation. In short, the gravitino mass will not affect inflation in our model. 


\section{Conclusion}

We have proposed a natural infaltion model based on string inspired SUGRA with gauged shift symmetry $\mathrm{U}(1)_{X}$. The matter fields are stabilized by F-terms, part of them obtain non-zero VEVs which break the anomalous $\mathrm{U}(1)_{X}$ spontaneously. They obtain masses several orders larger than the mass of inflaton, consequently the effect of vacuum energy from inflaton on field stabilization is seriously suppressed and ignorable. The modulusdependent FI term of $\mathrm{U}(1)_{X}$ plays a critical role in modulus stabilization. As the coupling between matter field and modulus is analytic, the D-term can be vanished in our model. Once the matter fields obtain non-zero VEVs, the real component of modulus $T$ is fixed by the D-term flatness. While the D-term is independent with the axionic component of $T$, therefore its cancellation has no effect on axion which remains light after modulus stabilization. The anomalous $\mathrm{U}(1)_{X}$ splits the masses of real and imaginary components of $T$. Such role of anomalous $\mathrm{U}(1)_{X}$ D-term on modulus stabilization has been studied in [26]. In the F-term moduli stabilization, usually the imaginary component of modulus obtains mass as large as the real component [24], the axion inflation cannot be realized. The reason is in F-term potential, the real and imaginary components of moduli couple with each other, it is highly non-trivial to split their masses at different scale so that one of them can play the role of inflaton while the other is frozen during inflation.

Potential for natural inflation is obtained from non-perturbative effect. Generally it is very difficult to get trans-Planckian axion decay constant, which is needed to fit with BICEP2 results. In our model, the axion decay constant linearly depends on the degree of condensation gauge group $\mathrm{SU}(n)$ and number of $\mathrm{U}(1)_{X}$ charged matter fields, therefore, the super-Planckian axion decay constant can be easily fulfilled by using large condensation gauge group and more $\mathrm{U}(1)_{X}$ charged fields.

\section{Acknowledgments}

The work of DVN was supported in part by the DOE grant DE-FG03-95-ER-40917. The work of TL is supported in part by by the Natural Science Foundation of China under grant numbers 10821504, 11075194, 11135003, and 11275246, and by the National Basic Research Program of China (973 Program) under grant number 2010CB833000.

Open Access. This article is distributed under the terms of the Creative Commons Attribution License (CC-BY 4.0), which permits any use, distribution and reproduction in any medium, provided the original author(s) and source are credited.

\section{References}

[1] BICEP2 collaboration, P.A.R. Ade et al., Detection of B-mode polarization at degree angular scales by BICEP2, Phys. Rev. Lett. 112 (2014) 241101 [arXiv:1403.3985] [INSPIRE].

[2] K. Freese, J.A. Frieman and A.V. Olinto, Natural inflation with pseudo-Nambu-Goldstone bosons, Phys. Rev. Lett. 65 (1990) 3233 [INSPIRE]. 
[3] F.C. Adams, J.R. Bond, K. Freese, J.A. Frieman and A.V. Olinto, Natural inflation: particle physics models, power law spectra for large scale structure and constraints from COBE, Phys. Rev. D 47 (1993) 426 [hep-ph/9207245] [INSPIRE].

[4] P. Svrček and E. Witten, Axions in string theory, JHEP 06 (2006) 051 [hep-th/0605206] [INSPIRE].

[5] K. Freese and W.H. Kinney, Natural inflation: consistency with cosmic microwave background observations of Planck and BICEPQ, arXiv: 1403.5277 [INSPIRE].

[6] K. Choi and J.E. Kim, Harmful axions in superstring models, Phys. Lett. B 154 (1985) 393 [Erratum ibid. 156B (1985) 452] [INSPIRE].

[7] T. Banks, M. Dine, P.J. Fox and E. Gorbatov, On the possibility of large axion decay constants, JCAP 06 (2003) 001 [hep-th/0303252] [INSPIRE].

[8] S. Dimopoulos, S. Kachru, J. McGreevy and J.G. Wacker, N-flation, JCAP 08 (2008) 003 [hep-th/0507205] [INSPIRE].

[9] A. R. Liddle, A. Mazumdar and F.E. Schunck, Assisted inflation, Phys. Rev. D 58 (1998) 061301 [astro-ph/9804177] [INSPIRE].

[10] J.E. Kim, H.P. Nilles and M. Peloso, Completing natural inflation, JCAP 01 (2005) 005 [hep-ph/0409138] [INSPIRE].

[11] K. Choi, H. Kim and S. Yun, Natural inflation with multiple sub-planckian axions, arXiv: 1404.6209 [INSPIRE].

[12] T. Higaki and F. Takahashi, Natural and multi-natural inflation in axion landscape, arXiv: 1404.6923 [INSPIRE].

[13] R. Kappl, S. Krippendorf and H.P. Nilles, Aligned natural inflation: monodromies of two axions, arXiv: 1404.7127 [INSPIRE].

[14] C. Long, L. McAllister and P. McGuirk, Aligned natural inflation in string theory, arXiv: 1404.7852 [INSPIRE].

[15] M. Cicoli, K. Dutta and A. Maharana, $N$-flation with hierarchically light axions in string compactifications, arXiv:1401.2579 [INSPIRE].

[16] F. Marchesano, G. Shiu and A.M. Uranga, F-term axion monodromy inflation, arXiv: 1404.3040 [INSPIRE].

[17] A. Hebecker, S.C. Kraus and L.T. Witkowski, D7-brane chaotic inflation, arXiv:1404.3711 [INSPIRE].

[18] R. Blumenhagen and E. Plauschinn, Towards universal axion inflation and reheating in string theory, arXiv:1404.3542 [INSPIRE].

[19] T.W. Grimm, Axion Inflation in F-theory, arXiv:1404.4268 [INSPIRE].

[20] R. Kallosh, A. Linde and B. Vercnocke, Natural inflation in supergravity and beyond, arXiv: 1404.6244 [INSPIRE].

[21] M. Arends et al., D7-brane moduli space in axion monodromy and fluxbrane inflation, arXiv: 1405.0283 [INSPIRE].

[22] S. Kachru, R. Kallosh, A.D. Linde and S.P. Trivedi, De Sitter vacua in string theory, Phys. Rev. D 68 (2003) 046005 [hep-th/0301240] [INSPIRE]. 
[23] S.B. Giddings, S. Kachru and J. Polchinski, Hierarchies from fluxes in string compactifications, Phys. Rev. D 66 (2002) 106006 [hep-th/0105097] [INSPIRE].

[24] R. Kallosh, On inflation in string theory, Lect. Notes Phys. 738 (2008) 119 [hep-th/0702059] [INSPIRE].

[25] T. Li, Z. Li and D.V. Nanopoulos, Aligned natural inflation and moduli stabilization from anomalous $\mathrm{U}(1)$ gauge symmetries, arXiv:1407.1819 [INSPIRE].

[26] T. Li, Z. Li and D.V. Nanopoulos, Chaotic inflation in no-scale supergravity with string inspired moduli stabilization, arXiv:1405.0197 [INSPIRE].

[27] T.R. Taylor, G. Veneziano and S. Yankielowicz, Supersymmetric QCD and its massless limit: an effective lagrangian analysis, Nucl. Phys. B 218 (1983) 493 [INSPIRE].

[28] D. Lüst and T.R. Taylor, Hidden sectors with hidden matter, Phys. Lett. B 253 (1991) 335 [INSPIRE].

[29] B. de Carlos, J.A. Casas and C. Muñoz, Massive hidden matter and gaugino condensation, Phys. Lett. B 263 (1991) 248 [inSPIRE].

[30] E. Dudas and S.K. Vempati, Large D-terms, hierarchical soft spectra and moduli stabilisation, Nucl. Phys. B 727 (2005) 139 [hep-th/0506172] [INSPIRE].

[31] G. Villadoro and F. Zwirner, De-Sitter vacua via consistent D-terms, Phys. Rev. Lett. 95 (2005) 231602 [hep-th/0508167] [INSPIRE].

[32] A. Achucarro, B. de Carlos, J.A. Casas and L. Doplicher, De Sitter vacua from uplifting D-terms in effective supergravities from realistic strings, JHEP 06 (2006) 014 [hep-th/0601190] [INSPIRE].

[33] Z. Lalak, G.G. Ross and S. Sarkar, Racetrack inflation and assisted moduli stabilisation, Nucl. Phys. B 766 (2007) 1 [hep-th/0503178] [INSPIRE].

[34] Z. Lalak, O.J. Eyton-Williams and R. Matyszkiewicz, F-term uplifting via consistent D-terms, JHEP 05 (2007) 085 [hep-th/0702026] [INSPIRE].

[35] P. Brax, A.-C. Davis, S.C. Davis, R. Jeannerot and M. Postma, D-term uplifted racetrack inflation, JCAP 01 (2008) 008 [arXiv:0710.4876] [INSPIRE].

[36] M. Dine, N. Seiberg and E. Witten, Fayet-Iliopoulos terms in string theory, Nucl. Phys. B 289 (1987) 589 [inSPIRE]. 